\title{
The Hydrogen Atom Fractal Spectra, the Missing Dark Energy of the Cosmos and Their Hardy Quantum Entanglement
}

\author{
Mohamed S. El Naschie \\ Department of Physics, Faculty of Science, University of Alexandria, Alexandria, Egypt \\ Email: Chaossf@aol.com
}

Received May 21, 2013; revised June 27, 2013; accepted July 5, 2013

Copyright (C) 2013 Mohamed S. El Naschie. This is an open access article distributed under the Creative Commons Attribution License, which permits unrestricted use, distribution, and reproduction in any medium, provided the original work is properly cited.

\begin{abstract}
In this letter, I outline the intimate connection between the fractal spectra of the exact solution of the hydrogen atom and the issue of the missing dark energy of the cosmos. A proposal for a dark energy reactor harnessing the dark energy of the Schrödinger wave via a quantum wave nondemolition measurement is also presented.
\end{abstract}

Keywords: Fractal Spectra; Dark Energy; Golden Mean; KAM Theorem; Quantum Entanglement; Special Relativity

The spectrum of the hydrogen atom was found in 2006 by V. Petruševski to harbor the golden mean for which the discoverer could not give any deep rational explanation [1,2]. On the other hand since the 1988 Physics Review Article of C. DeVito and W. Little [3], it was known that the spectral lines of the hydrogen atom [4] are a kind of Cantor set fractal but they could not say exactly what form of fractal set it is nor from where this fractality stems $[3,5]$.

The present work reports on an important discovery which links the above mentioned two papers together and gives a clear cut rational explanation for the fractality and the golden mean of the hydrogen atom spectra and moves from there to explaining dark energy [4,5-7]. It is simply the involvement of random Cantor sets $[8,9]$ in the structure of the spectra on the very fundamental level of Hardy type quantum entanglement in physics [5-10] which leads to the golden mean and the random fractal appearance [5-14]. A random Cantor set possesses with a probability 1, a Hausdorff dimension equal to the golden mean $(\sqrt{5}+1) / 2$ [11-14]. This is actually a theorem by Mauldin and Williams [8-12]. Furthermore the Hardy type probability of quantum entanglement of two particles is equal to the golden mean to the power of 5, i.e. $\mathrm{P}($ Hardy $)=\phi^{5}=9.01699 \%$ [5-7]. This particular result was confirmed in various sophisticated experiments $[5,10]$ so that we could state confidently that it strongly suggests that quantum spacetime is a Cantorian fractal as advocated in various fractal-Cantorian spacetime theories as well as the theory of non-commutative spaces [14] as applied to Penrose fractal tiling [8-10,13,14]. The concerted action of all these facts and elements leads us to recognize the golden mean random Cantor set nature of the spectra of the hydrogen atom. For instance, in certain cases (labeled 25 in Ref. [4], p. 192), which will be discussed in detail in a forthcoming paper, replacing the Bohr radius in the corresponding probability density [4] with the isomorphic length $\left(4+\phi^{3}\right) / 2$ of Penrose tiling one obtains Hardy's probability of quantum entanglement. Consequently the present analysis adds more weight to the assertion of the proponents of fractal-Cantorian spacetime that quantum spacetime is indeed a Cantorian $[13,14]$ fractal with a topological dimension [6-9] of exactly $D_{t}=4$ and a likewise an exact Hausdorff dimension given by $[6-9,13]$

$$
\begin{aligned}
D_{H} & =4+\frac{1}{4+\frac{1}{4+\cdots}} \\
& =4+\phi^{3} \\
& =4.23606799
\end{aligned}
$$

where $\phi=0.6183398$ is the golden mean [1-15].

Quantum entanglement is not only part and parcel of an old basic problem of quantum theory such as the hydrogen atom but also at an unimaginably deeper level covering the entire universe being the key to the missing 
dark energy of the cosmos [16]. In short, it turns out that the famous relativity formula $E=m c^{2}$ relating mass (m) to energy (E) via the speed of light (c) does not distinguish between measurable real ordinary energy $\mathrm{E}(\mathrm{O})$ and missing dark energy of the cosmos E(D) which cannot be detected or measured directly using any of present day technology $[17,18]$. The simple explanation for this unparalleled challenge to the foundations of modern theoretical physics and cosmology is again intimately connected to Hardy's quantum entanglement and consequently to random Cantor sets and their golden mean Hausdorff dimensions. Since at the quantum resolution level, spacetime is made up of totally disjoint random Cantor sets with an infinite number of graded dimensions, the probability of quantum entanglement is governed by the zero measure of this Cantorian space and is given generally by $P=\left(\phi^{n}\right)\left(\phi^{3}\right)=\phi^{n+3}$ where $n$ is the number of particles [5,7]. For two particles, one finds $P=\phi^{5}$ which is the theoretically and experimentally confirmed Hardy quantum probability [5-7]. Intersecting the three fundamental equations of physics, namely Newton's kinetic energy $E_{N}=\frac{1}{2} m v^{2}$ where $v$ is the velocity, Einstein's equation $E=m c^{2}$ and $P=\phi^{5}$ one finds a quantum relativity energy-mass formula

$$
E_{Q R}=\left(\frac{1}{2} m\right)(v \rightarrow c)^{2}\left(\phi^{5}\right)=\left(\phi^{5} / 2\right) m c^{2} \simeq m c^{2} / 22 .
$$

This is exactly the energy density found via accurate cosmic measurements COBE and WMAP which amount to only $1 / 22 \cong 4.5 \%$ of what $\mathrm{E}$ (Einstein) predicted [10], [16-19]. The rest namely $(1-(1 / 22)) \simeq 95.5 \%$ is the presumed missing dark energy of the Universe. It then turned out that ordinary energy i.e. the measurable $4.5 \%$ is simply the energy of the quantum particle face of the particle wave duality of quantum mechanics $[7,10]$ while the missing $95.5 \%$ dark energy $\mathrm{E}(\mathrm{D})$ is the energy of the other face of the duality, namely the quantum wave $[10,14,18]$. Since the quantum particle is modeled by a five dimensional zero set and the wave is modeled by a five dimensional empty set we have here a zero setempty set duality [19].

Together $E(c)=m c^{2} / 22$ of the particle and $E(D)=5\left(\phi^{2} / 2\right)\left(m c^{2}\right) \simeq m c^{2}(21 / 22)$ of the wave add up to a total exactly equal to Einstein's energy [16-19]:

$$
E(\text { total })=\left[\left(\phi^{5} / 2\right)+\left(5 \phi^{2} / 2\right)\right]\left(m c^{2}\right)=m c^{2} .
$$

Two major conclusions follow after the above. First, Einstein's $E=m c^{2}$ is correct but completely blind to the distinction between dark energy and ordinary energy. Second, since measurement collapses the quantum wave, it is natural that our present day technology cannot detect dark energy [10,16-18]. From the above we can see fu- ture technological research going towards developing quantum nondemolition measurement instruments and nuclear dark energy reactors. None the less it is vital to understand that we may have a wavy spacetime at the quantum resolution simulating a quantum wave of a knot in the fabric of spacetime. Consequently harvesting the quantum wave may be harvesting the real vacuum modeled by the empty set, similar to what KAM theorem implies [8].

Finally we note that $\mathrm{E}(0)$ and $\mathrm{E}(\mathrm{D})$ could be readily found from the ground energy state of the hydrogen atom $E=\frac{1}{2} m c^{2} \alpha^{2}$ where $\alpha=1 / 137 \quad[4,20]$ by running $\alpha$ as a function of energy as in quantum field theory and replacing $\alpha^{2}$ by $\phi^{2} \phi^{3}=\phi^{5}$ so that we find

$E=\frac{1}{2} m c^{2} \phi^{5}=m c^{2} / 22$.

\section{REFERENCES}

[1] V. M. Petruševski, "The H-Atom and the Golden Ratio: A Possible Link," Journal of Chemical Education, Vol. 83, No. 1, 2006, p. 40.

[2] V. M. Petruševski, "The First Excited State of the Hydrogen Atom and the Golden Ratio: A Link or a Mere Coincidence?” Bulletin of the Chemists and Technologists of Macedonia, Vol. 25, No. 1, 2006, pp. 61-63.

[3] C. L. Devito and W. A. Little, "Fractal Sets Associated with Function: The Spectral Lines of Hydrogen,” Physical Review A, Vol. 38, No. 12, 1988, pp. 6362-6364. doi:10.1103/PhysRevA.38.6362

[4] A. C. Phillips, "Introduction to Quantum Mechanics," John Wiley \& Sons Ltd., Chichester, 2003.

[5] M. S. El Naschie, "Quantum Entanglement as a Consequence of a Cantorian Micro Spacetime Geometry," Journal of Quantum Information Science, Vol. 1, No. 2, 2011, pp. 50-53. doi:10.4236/jqis.2011.12007

[6] J.-H. He, et al., "Quantum Golden Mean Entanglement Test as the Signature of the Fractality of Micro Spacetime,” Nonlinear Science Letters B, Vol. 1, No. 2, 2011, pp. 45-50.

[7] L. Hardy, "Nonlocality of Two Particles without Inequalities for Almost All Entangled States," Physical Review Letters, Vol. 71, No. 11, 1993, pp. 1665-1668. doi:10.1103/PhysRevLett.71.1665

[8] M. S. El Naschie, "A Review of E-Infinity Theory and the Mass Spectrum of High Energy Particle Physics," Chaos, Solitons \& Fractals, Vol. 19, No. 1, 2004, pp. 209-236. doi:10.1016/S0960-0779(03)00278-9

[9] M. S. El Naschie, "The Theory of Cantorian Spacetime and High Energy Particle Physics (An Informal Review)," Chaos, Solitons \& Fractals, Vol. 41, No. 5, 2009, pp. 2635-2646. doi:10.1016/j.chaos.2008.09.059

[10] R. Penrose, “The Road to Reality,” Jonathan Cape, London, 2004.

[11] R. Mauldin and S. Williams, "Random Recursive Con- 
structions: Asymptotic Geometries and Topological Properties," Transactions of the American Mathematical Society, Vol. 295, No.1. 1986, pp. 325-346. doi:10.1090/S0002-9947-1986-0831202-5

[12] R. Mauldin, "On the Hausdorff Dimension of Graphs and Recursive Object,” In: G. Mayer-Kress, Ed., Dimension and Entropies in Chaotic Systems, Springer, Berlin, 1986, pp. 28-33.

[13] L. Marek-Crnjac, "The Hausdorff Dimension of the Penrose Universe," Physics Research International, Vol. 2011, 2011, pp. 1-4.

[14] A. Connes, "Noncommutative Geometry," Academic Press, San Diego, 1994.

[15] M. Gardener, "Penrose Tiles to Trapdoor Ciphers,” W.H. Freeman, New York, 1989.

[16] L. Amendola and S. Tsujikawa, "Dark Energy: Theory and Observations," Cambridge University Press, Cam- bridge, 2010.

[17] L. Marek-Crnjac, et al., "Chaotic Fractals at the Root of Relativistic Quantum Physics and Cosmology,” International Journal of Modern Nonlinear Theory and Application, Vol. 2, No. 1A, 2013, pp. 78-88. doi:10.4236/ijmnta.2013.21A010

[18] M. S. El Naschie, “A Resolution of Cosmic Dark Energy via a Quantum Entanglement Relativity Theory,” Journal of Quantum Information Science, Vol. 3, No. 1, 2013, pp. 23-26. doi:10.4236/jqis.2013.31006

[19] M. S. El Naschie, “Topological-Geometrical and Physical Interpretation of the Dark Energy of the Cosmos as a 'Halo' Energy of the Schrödinger Quantum Wave,” Journal of Modern Physics, Vol. 4, No. 5, 2013, pp. 591-596. doi:10.4236/jmp.2013.45084

[20] S. Brandt and H. Dahmen, "The Picture Book of Quantum Mechanics,” Springer, New York, 1995, pp. 237-238. 